\title{
Taxi Ride Based on Voice Alert System
}

\author{
Swetha.R.V ${ }^{1}$, Akshaya.R ${ }^{2}$, Sharmila.A ${ }^{3}$, Sri Lekha.P ${ }^{4}$, Mrs.K.Lalitha ${ }^{5}$ \\ 1, 2,3,4 Under Graduate Student, Department of Information Technology, Panimalar Engineering College, Chennai, TamilNadu, \\ India \\ ${ }^{5}$ Asst.Professor, Department of Information Technology, Panimalar Engineering College, Chennai, TamilNadu, India
}

\begin{abstract}
Previous applications are not well efficient as they provide voice alerts only for the tagged locations whereas in this application voice alerts are given for any location to the user, in terms of time and distance. It would be useful even when we are out of home language/place. It is an integration of Google Maps and voice Alert. In this application, once the cab is booked it gives voice alert to the user about its current location and time it would reach the user's location. This application also allows the user to know about the required nearby locations. The user can also pin any new landmark for their personal usage into Google maps.
\end{abstract}

Keywords — taxi ride, voice alert, google maps.

\section{INTRODUCTION}

Recent studies reveal that the utilization of location based services has increased with the boost in the usage of the mobile devices. Since the current location of users can be detected with the embedded sensors in the mobile devices, employing the context information to notify the users became possible. Also the demand for taxis in peak time is much higher. Increasing the taxi count is not a better idea, instead utilizing the available resources in an effective way will be a good solution for this problem.

A real time taxi sharing application is been introduced which will ping the passengers as far as possible, in a time and cost efficient way both to passengers and taxis. This application excels in privacy and security related preferences. This type of car Pooling makes use of three recent technological advancements such as GPS navigation devices to determine the driver's route and arrange the shared ride, SMARTPHONES and Internet Connectivity.

Voice Alert of the cab's current location will be given to the user in terms of time and distance. This application displays and provides voice recommendation of time, distance and also provides route to the user's required nearby places for the specified category such as ATM, Restaurants, Gas stations, etc. New locations can also be marked by the user privately for future references through this application.

\section{EXISTING SYSTEM}

In traditional location based reminder system, the user tags the location using the applications such as Google maps, Foursquare or via the embedded sensors of the Android device. Then, the user creates reminders for the tagged location and when they get closer to the location the system notifies the user. Main purpose of this application is to allow users to create reminders based on the location besides time and to notify users with those reminders automatically.But the traditional location based reminders may not be suitable if the users expect to be notified when they approach to a specific location.

\section{PROPOSED SYSTEM}

Our system is proposed to establish software application that would be utilized by people to prepare for the journey and get the alert throughout the journey. This application will immediately alert the user with voice whenever the cab starts its journey from its location.

Main function of this application is to permit users to develop suggestions based on the requirements to get aware of nearby places(ATM, hotels, bunks, etc.,) and to inform them about its location and the time taken to reach it instantly using voice alert system. This application allows the user to even pin any new landmark for their personal usage into Google maps.

This GPS (Global Positioning System) based application will trigger the voice whenever device reaches a specified location. Application will get the Latitude and Longitude points on the device and will alert about the current location on Map. Application also can be merged with some information like, location of the nearest restaurants, hotels, shopping malls, etc. 


\section{ARCHITECTURE DIAGRAM}

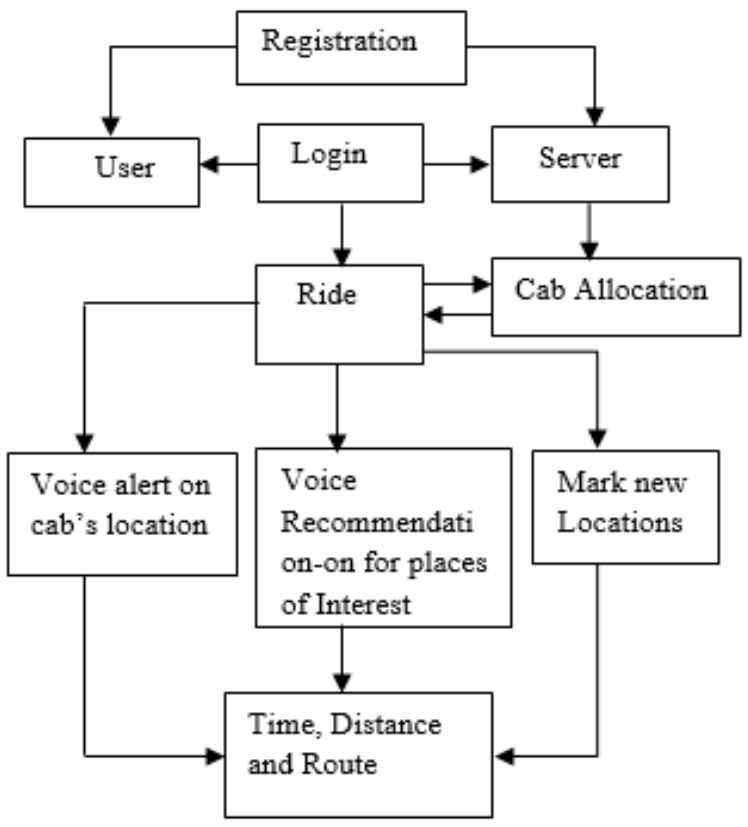

Fig.1: Architecture Diagram

\section{SYSTEM ARCHITECTURE}

The Fig.1 shows the system architecture which is described using three units which are Registration and Login, Allocation of Cabs and Ride. The workflow of the Architecture has been explained below:

\subsection{REGISTRATION AND LOGIN}

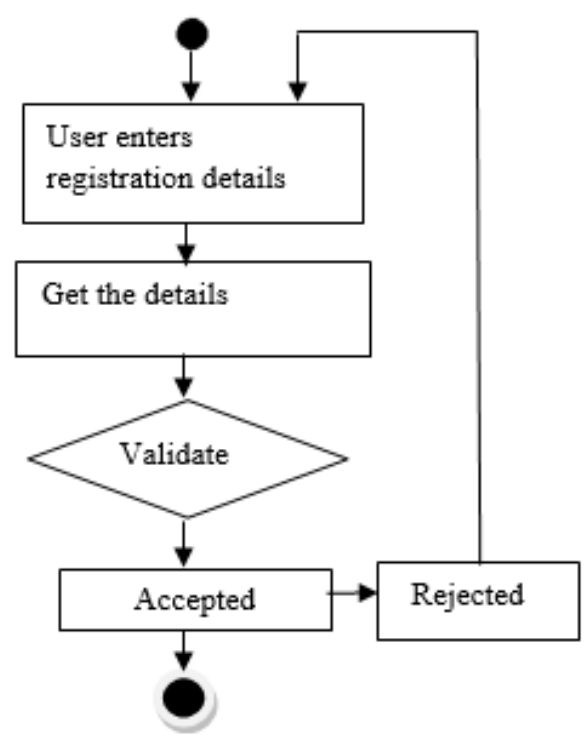

Fig.2 : Registration

These Fig. 2 and Fig. 3 explains that the user registers their account into the application to initiate the ride for the first time. All registration details are stored in SQL which can also be viewed by the server. The registered users can just login into the application to start their ride and can also edit their profile if needed. One needs to enter the source and destination details in order to book a cab.

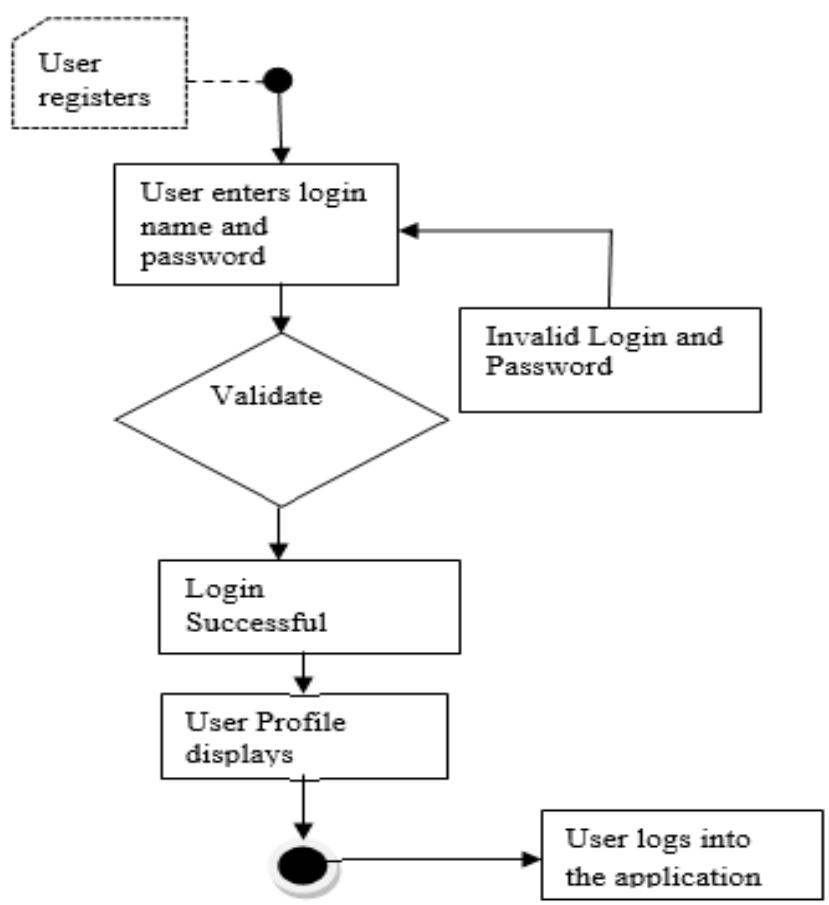

Fig.3:Login

\subsection{ALLOCATION OF CABS}

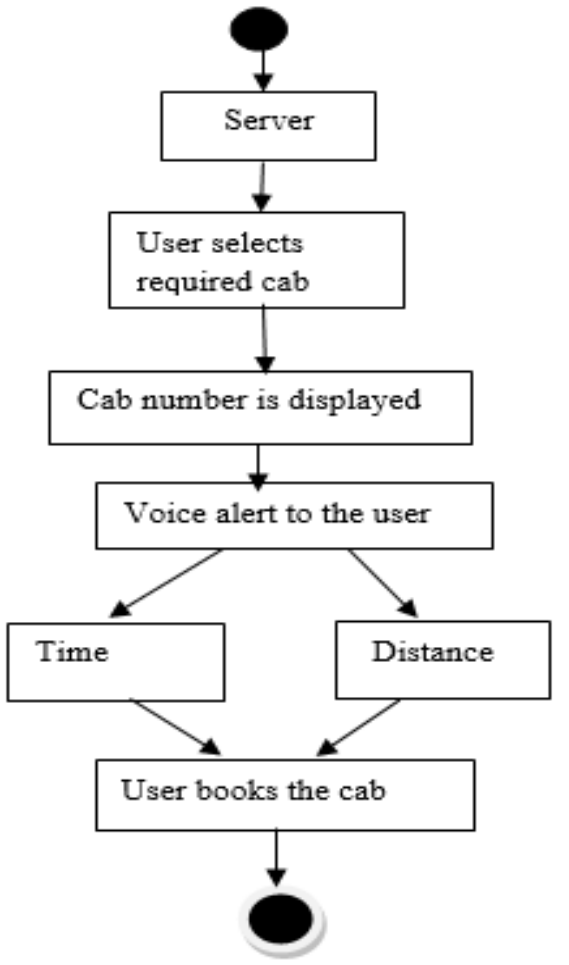

Fig.4: Cab allocation 
The Fig.4 explains that once the User requests for the ride, the Server searches for the available cab nearby to the user and allocates the one closest to the user. The User is notified with the vehicle number, cab's current location and the time it takes to reach the source.

\subsection{RIDE}

The ride is followed by three modules namely,

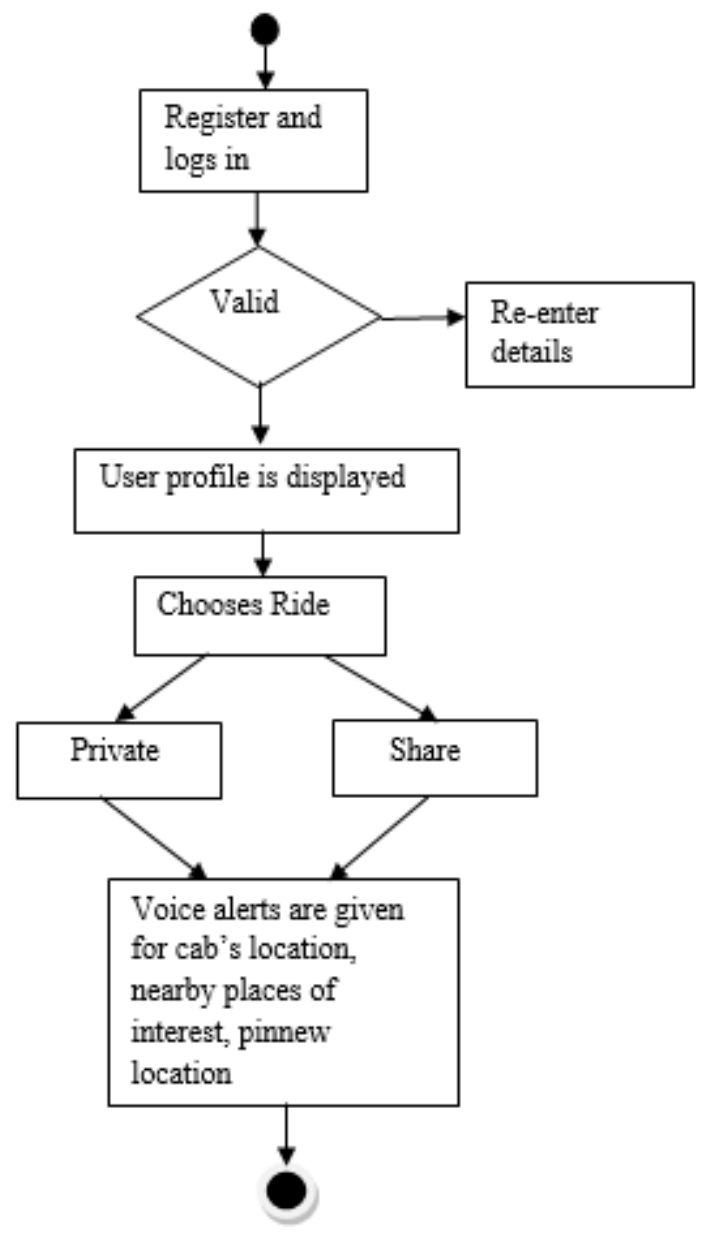

Fig.5:Ride

\section{(i)Voice Alert of the Cab's Location:}

The user receives a voice alert of cab's current location which has not yet arrived to the source. The voice alert describes the distance and the time it would take to reach the user. The user can also check for the cab's location frequently, if needed.

\section{(ii)Nearby places of Interest:}

The user can search for the nearby places under the specified category and in turn receives a voice recommendation for the nearest one. The Time, Distance and the Route to reach the suggested place from the user's location through voice.

\section{(iii)Pin new location:}

The user can mark any newly found locations privately, so that they can use for the next time in order to reduce the search process again.

\section{CONCLUSION}

Hence we developed an efficient ride sharing application which enables the user to travel to their destinations with ease and fair subject to time, capacity and monetary constraints for both user and driver. In addition, we have also included voice recommendation (time,distance) for the required nearby places for the specified category along with the route. Moreover, the user can also pin new locations privately for their future references.

\section{REFERENCES}

[1] Mr.Swapnil S. Nate, Mr.Pravin S. Navele, Mr.Vikas B. Mote, Prof. Laxman S. Naik: Smart Reminder Application with GPS System, In: International Research Journal of Engineering and Technology. (2016)

[2] ItirOnal, Ali MertErtugrul, RemindMe: An Enhanced Mobile Location-Based Reminder Application, In: Future Internet of Things and Cloud (FiCloud), International Conference on Multimedia.(2014)

[3] Li Y.,GuoA.,Liu S.,Gao Y. and Zheng Y.: A Location Based Reminder System for Advertisement, In: Proceedings of the International Conference on Multimedia, 1501-1502 (2010)

[4] Persson,P., Espinoza F., Fagerberg P., Sandin A. and Coster R: GeoNotes: A Location-Based Information System for Public Spaces, Designing Information Spaces: The Social Navigation Approach, 151- 173 (2003)

[5] Dey A. K. and Abowd G.D.: CyberMinder: A Context-Aware System for Supporting Reminders, In: Proceedings of the 2nd International Symposiumon Handheld and Ubiquitous Computing (HUC2K)(2000) 